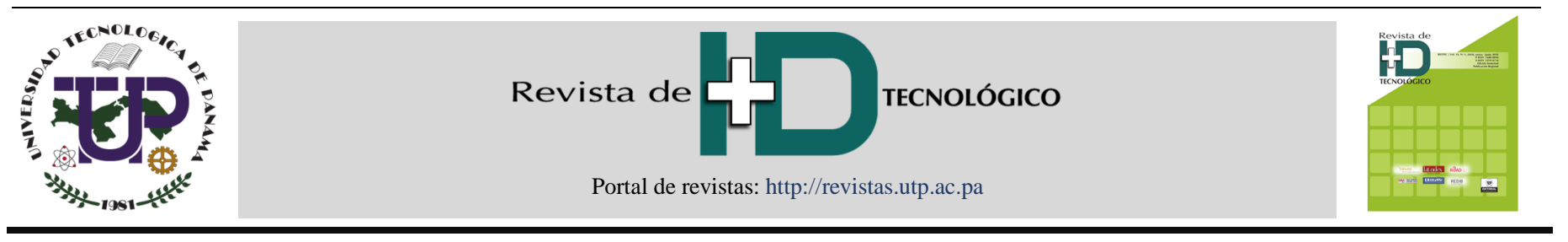

\title{
Método fotogramétrico pragmático para medir desplazamientos en puentes
}

\section{Pragmatic photogrammetric method to measure displacements in bridges}

\author{
Noé A. Serrano ${ }^{1 *}$, Brayan A. Zuñiga ${ }^{2}$, José M. Gallardo ${ }^{3}$ \\ ${ }^{1,2,3}$ Grupo de investigación: Salud Estructural de Puentes, ${ }^{1,2,3}$ Universidad Tecnológica de Panamá, Panamá \\ *Autor de correspondencia: jose.gallardo@utp.ac.pa
}

\begin{abstract}
RESUMEN-En este artículo se presenta un método fotogramétrico para medir desplazamientos en puentes, utilizando una cámara digital y software de análisis de videos, como herramienta para la evaluación estructural de puentes. Este método permite registrar el desplazamiento vertical de los puentes, el cual es el parámetro más relevante para estimar la capacidad de los puentes, tanto durante pruebas de carga estáticas como durante el tráfico vehicular. El método propuesto es económico y sencillo, permite alta frecuencia de lectura $(60 \mathrm{~Hz})$, y ofrece medidas confiables, con errores de medición en el orden de $0.1 \mathrm{~mm}$ al medir desplazamientos y $0.01 \mathrm{~Hz}$ al estimar frecuencias de vibración. Este método es atractivo al compararlo con alternativas mayormente utilizadas actualmente, como los deformímetros de alta precisión, que requieren apoyarse en estructuras temporales costosas, o como los equipos de medición topográfica que cuentan con precisiones en el orden de $1 \mathrm{~mm}$. El método fue validado mediante experimentación en especímenes de madera. Se demostró su uso práctico al implementarlo en el monitoreo de desplazamientos verticales, y la frecuencia de vibración principal, de cuatro puentes bajo la acción del tráfico regular.
\end{abstract}

Palabras claves-Puentes, desplazamiento, aceleración, frecuencia, vibración, fotogrametría.

\begin{abstract}
This paper presents a photogrammetric method to measure displacement in bridges, using a digital camera and video analysis software, as a tool for the structural assessment of bridges. This method allows to record the vertical displacement of the bridges, which is the most important parameter to estimate the capacity of the bridges, both during static load tests and during vehicular traffic. The proposed method is economical and simple; allows high frequency of reading $(60 \mathrm{~Hz})$, and provides reliable measurements, with measurement errors in the order of $0.1 \mathrm{~mm}$ when measuring displacement and $0.01 \mathrm{~Hz}$ when estimating vibration frequencies. This system is attractive when compared to alternatives most commonly used today, such as high precision deformimeter, which requires support in costly temporary structures, or as topographic measuring equipment that have precisions in the order of $1 \mathrm{~mm}$. The method was validated by experimentation on wood specimens. In its practical application, it was used to measure the vertical displacements, and the main vibration frequency, of four bridges under the action of regular traffic.
\end{abstract}

Keywords-Bridges, displacement, acceleration, frequency, vibration, photogrammetry.

\section{Introducción}

Los puentes se deterioran con el paso del tiempo, y es de vital importancia conocer su condición estructural, ya que el deterioro de los puentes en servicio puede generar desplazamientos o vibraciones excesivas, causando molestias en los usuarios y agudizando daños en elementos estructurales y no estructurales, o en el peor de los casos el colapso de la estructura. En las últimas décadas los métodos no destructivos para la detección de daños en estructuras civiles han ganado especial atención por parte de investigadores de todo el mundo, debido a los avances y disponibilidad de las tecnologías de instrumentación y de procesamiento digital [1].

La prueba de carga es el método más aceptado para estimar la capacidad de los puentes. El desplazamiento vertical es uno de los parámetros más relevantes a medir durante las pruebas de carga. Algunas de las limitantes o dificultades relacionadas al uso de varios de los sistemas utilizados para medir el desplazamiento vertical de puentes son: la dificultad de instalación de sensores como las galgas extensiométricas en puentes existentes, la precisión en el orden de $1 \mathrm{~mm}$ que ofrecen las estaciones totales es relativamente baja para puentes cortos, y la dificultad de la instrumentación cuando se utilizan deformímetros (o comparadores).

Por otro lado, el impacto que genera el cierre del tráfico durante las pruebas de carga, hace deseable contar con métodos que permitan la medición del desplazamiento dinámico de puentes -información que se puede utilizar para estimar preliminarmente la salud estructural del puente- sin cerrar el tráfico [2]. Las

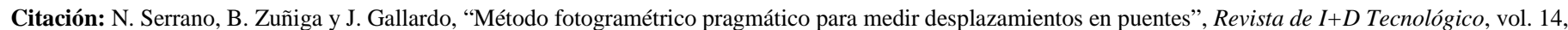
n. ${ }^{\circ}$ 1, pp. 29-40, Jun. 2018.

Tipo de artículo: Original. Recibido: 27 de octubre de 2017. Recibido con correcciones: 31 de octubre de 2017 . Aceptado: 11 de abril de 2018.

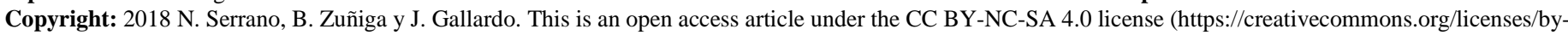
nc-sa/4.0/). 
vibraciones son mayormente registradas por medio de acelerómetros; actualmente están disponibles acelerómetros económicos, cuya data ha sido utilizada exitosamente para determinar las frecuencias naturales de vibración de la estructura, con el fin de detectar cambios que pudieran estar relacionados con deterioro estructural [3]. Sin embargo, este método no ofrece una medida confiable de uno de los parámetros más relevantes, el desplazamiento, ya que la doble integración de la data obtenida de los acelerómetros económicos genera errores considerables. En este sentido se han realizado varias investigaciones que proponen métodos para la medición de desplazamientos dinámicos $[4,5,6,7,8,9,10,11,12,13]$.

En esta investigación se propone un método económico, de fácil instrumentación, con alta precisión y alta frecuencia de lectura, para la medición de desplazamientos en puentes. El método es útil para el monitoreo bajo tráfico regular, o para mediciones durante pruebas de carga dinámicas o estáticas. El método utiliza una cámara digital de alta resolución, una referencia sobre la estructura del puente y un programa computacional para procesar el video.

El método fue validado mediante pruebas estáticas sobre un espécimen de madera, en la cual se comparó los resultados del nuevo método versus los resultados de un deformímetro de alta precisión, obteniendo diferencias menores a $0.1 \mathrm{~mm}$. Adicionalmente, se realizó pruebas dinámicas en donde se comparó las frecuencias de vibración obtenidas utilizando el método propuesto versus los resultados de la data captada por acelerómetros, obteniendo diferencias en el orden de 0.1 $\mathrm{Hz}$.

Para ilustrar el uso práctico del método propuesto, se implementó sobre 4 puentes vehiculares, se monitoreó los desplazamientos verticales en el centro de los puentes bajo la acción del tráfico regular; así se obtuvo el desplazamiento máximo y la frecuencia de vibración de los puentes.

\section{Revisión literaria}

La deformación a la que se somete un puente durante las pruebas de carga, debe cumplir con la normativa AASHTO LRFD, la cual establece una deflexión máxima permitida de L/800, para el paso de carga vehicular en puentes de acero y/o concreto; donde L es la longitud del claro [14]. Determinar con gran precisión los desplazamientos en los puentes, ha sido un problema difícil de resolver en la ingeniería civil, por esta razón se han realizado muchas investigaciones sobre este tema y se han aplicado numerosos métodos. Entre los equipos utilizados en los métodos más destacados están:

- Deformímetro (comparador) [15]: este tipo de instrumentos es preciso y de alta resolución, hasta de $0.001 \mathrm{~mm}$; sin embargo, requieren instalarse en una plataforma que se apoye en terreno firme, lo cual puede ser extremadamente costoso o impráctico.

- Galgas extensiométricas [16]: se tienen que colocar en la estructura del puente y son afectadas por las condiciones ambientales.

- GPS vía satélite [17]: este equipo se ve afectado por el clima, y permite una frecuencia de medición muy baja.

- La estación total [13]: este dispositivo tiene precisión de aproximadamente $1 \mathrm{~mm}$, y ha sido ampliamente utilizado en el control de desplazamiento en ingeniería. Su baja frecuencia de medición dificulta su uso durante el tráfico vehicular normal.

- Escaneo Láser 3D [18]: este método puede crear modelos complejos en 3D, y ha sido utilizado para estudiar el desplazamiento de estructuras masivas; sin embargo, la frecuencia de lectura es baja, y el post-tratamiento de datos es complicado.

En resumen, en la actualidad existe una amplia variedad de métodos para medir desplazamientos de estructuras, cada uno de ellos con sus ventajas y limitaciones. En los últimos años se han desarrollado nuevos métodos, para la detección de desplazamientos en puentes, basados en técnicas de fotogrametría para procesar videos, que contienen información del desplazamiento del puente.

Varios estudios han utilizado cámaras digitales y puntos marcados en las estructuras de interés $[4,6,9$, 11]; las cámaras se colocan fuera de la estructura, desde donde se graban los desplazamientos de los puntos. Técnicas de fotogrametría se utilizan para determinar el movimiento de los puntos en el video, el movimiento de los puntos representa el desplazamiento de la estructura. También, se ha demostrado que se puede obtener una alta precisión en pruebas laboratorio, obteniendo una precisión media de $0.1 \mathrm{~mm}$ [6]. En una prueba de carga sobre un puente, se estimó una desviación estándar del orden de $0.1-0.2 \mathrm{~mm}$, comparando los resultados del 
nuevo método con sensores electrónicos de medición de desplazamiento [4].

Algunos estudios han utilizado laser y cámaras digitales, junto con técnicas de fotogrametría para medir desplazamientos $[5,7,8,10]$. Este método consiste en la colocación del láser en el punto de interés de la estructura y proyectar el haz de luz láser a una pantalla, donde se graba con la cámara digital. El movimiento del láser en la pantalla está relacionado con el desplazamiento de la estructura. Utilizando este método se han realizado pruebas de comparación con transductores de desplazamiento variable lineal (LVDT) obteniendo resultados similares [7]. En laboratorio se han realizado pruebas estáticas, con errores de $0.04 \mathrm{~mm}$ y reconocimiento de cambio de distancia mínima de $0.02 \mathrm{~mm}$ [10]. Adicionalmente, este método ha sido implementado en puentes peatonales [8].

Estos nuevos métodos basados en técnicas de fotogrametría para el procesamiento digital de imágenes están recibiendo considerable atención, ya que representa un avance en el área de monitoreo estructural, porque pueden llegar a ser aplicables en la medición de desplazamientos de grandes estructuras y a grandes distancias, gracias al avance en la tecnología de las cámaras digitales, que actualmente cuentan con un gran alcance, gran resolución en las imágenes y altas velocidades de captación de imágenes. Los resultados experimentales de investigaciones anteriores muestran que este sistema es factible, de fácil instalación, fácil manejo, bajo costo, alta frecuencia de adquisición de imágenes y de gran precisión para determinar el desplazamiento en estructuras como puentes. Por lo cual en esta investigación desarrollamos una variante de este novedoso método fotogramétrico, para el seguimiento de un punto sobre la estructura de puentes, y así obtener los desplazamientos verticales.

Para determinar el método fotogramétrico más conveniente en este estudio implementaremos en laboratorio el uso de las dos variantes más usadas de este método:

- Proyección de un láser colocado en el punto de interés del puente (Laser + Cámara).

- Grabación de un punto colocado sobre la estructura (Punto + Cámara).

\section{Método experimental}

Con la finalidad de medir desplazamientos en los puentes se desarrolló un programa experimental que utiliza las técnicas de procesamiento de video digital para el seguimiento de un punto de referencia colocado sobre la estructura de los puentes. El método se aplicó en laboratorio para verificar su desempeño, y en campo para verificar su uso práctico.

Durante pruebas en laboratorio se midió deformaciones en un espécimen de madera, durante prueba estática y dinámica. El espécimen se instrumentó con el sistema requerido para el método nuevo y, adicionalmente, con un deformímetro para medir desplazamiento durante la prueba estática y acelerómetro para obtener el registro de aceleración durante la prueba dinámica. Los resultados de los sensores de desplazamiento y aceleración servirán como referencia para comparar con los resultados del nuevo método.

La prueba en campo se realizó en 4 puentes vehiculares de estructura de concreto con vigas tipo ASSTHO, con longitudes entre 35 y 42 m (ver tabla 1), ubicados en la carretera panamericana entre el distrito de Boquerón y el distrito de David, en la provincia de Chiriquí (ver figura 1). Cada luz (o claro) de los puentes se identificó con un código basado en el posicionamiento geográfico del centro de la misma; el código cuenta con tres partes: primero la letra L (en referencia a la palabra "Luz"), luego la latitud UTM y finalmente la longitud UTM, ambas dentro de la zona 17. En estos puentes se utilizó el método de punto+cámara para registrar desplazamientos verticales dinámicos y acelerómetro para monitorear aceleraciones producto del tráfico vehicular regular.

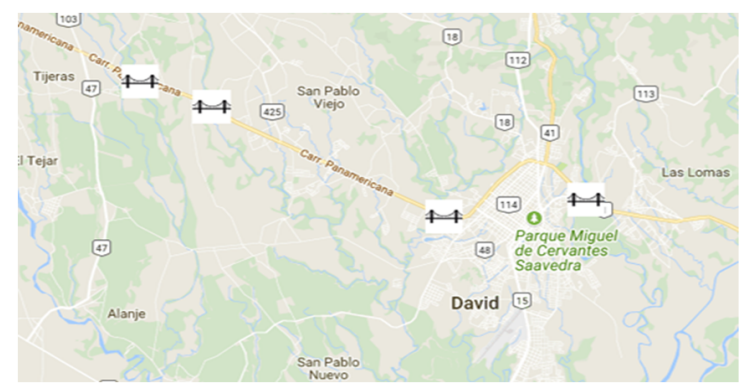

Figura 1. Mapa con los puentes a instrumentar en la provincia de Chiriquí. 
Tabla 1. Puentes instrumentados

\begin{tabular}{|c|c|c|c|}
\hline Puente sobre: & $\begin{array}{c}\text { Código } \\
\text { (coordenada } \\
\text { UTM, zona 17) }\end{array}$ & $\begin{array}{c}\text { Luz } \\
\text { (m) }\end{array}$ & $\begin{array}{c}\text { Viga } \\
\text { AASHTO } \\
\text { tipo: }\end{array}$ \\
\hline $\begin{array}{c}\text { Río Chirigagua } \\
\text { aguas abajo }\end{array}$ & $\begin{array}{c}\text { L-330300E- } \\
936810 \mathrm{~N}\end{array}$ & 35.4 & IV \\
\hline $\begin{array}{c}\text { Río Platanal } \\
\text { aguas abajo }\end{array}$ & $\begin{array}{c}\text { L-332610E- } \\
935900 \mathrm{~N}\end{array}$ & 38.6 & $\mathrm{IV}$ \\
\hline $\begin{array}{c}\text { Quebrada San } \\
\text { Cristóbal } \\
\text { aguas abajo }\end{array}$ & $\begin{array}{c}\text { L-340130E- } \\
931970 \mathrm{~N}\end{array}$ & 35.6 & $\mathrm{IV}$ \\
\hline $\begin{array}{c}\text { Río David aguas } \\
\text { abajo (Luz 1) }\end{array}$ & $\begin{array}{c}\text { L-344740E- } \\
932560 \mathrm{~N}\end{array}$ & 42.0 & $\mathrm{~V}$ \\
\hline $\begin{array}{c}\text { Río David aguas } \\
\text { abajo (Luz 2 ) }\end{array}$ & $\begin{array}{c}\text { L-344760E- } \\
932530 \mathrm{~N}\end{array}$ & 42.0 & $\mathrm{~V}$ \\
\hline
\end{tabular}

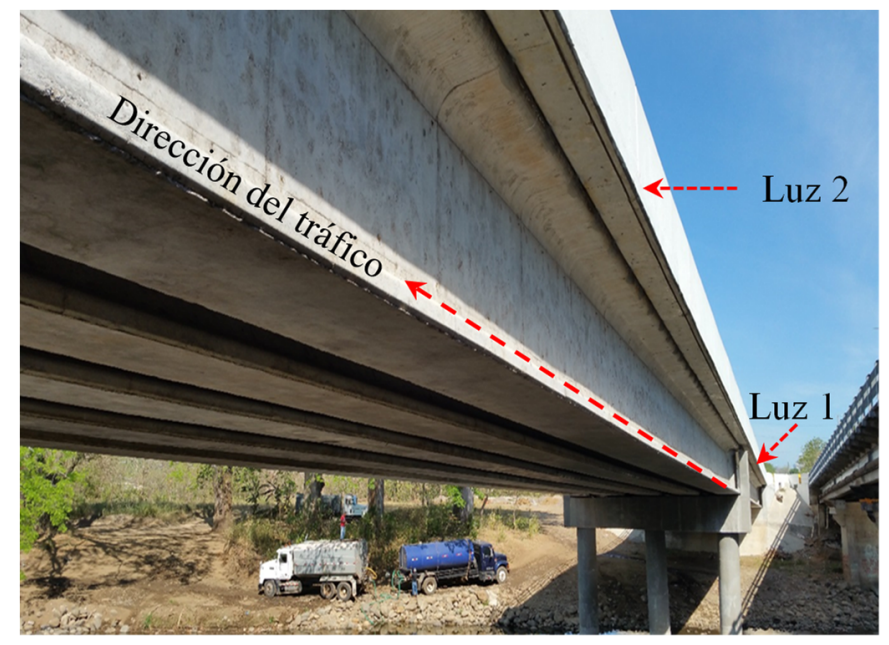

Figura 2. Vista del puente sobre el Río David aguas abajo.

\subsection{Equipo}

Para la medición de desplazamientos y aceleraciones se utilizaron los siguientes equipos:

- Cámara digital: modelo Nikon Coolpix P900. Se eligió esta cámara por su costo relativamente bajo, ya que es una cámara de uso comercial, por su capacidad de enfocar y obtener video de alta resolución a distancias de varias decenas de metros. Sus principales características son: distancia focal de 4.3 a $357 \mathrm{~mm}$, y grabación de video a $30 \mathrm{fps}$ y 60 fps (equivalente a $60 \mathrm{~Hz}$ ) a una resolución de 1920x1080 pixeles.

- Pantalla de referencia: pantalla blanca con un punto marcado en ella, y una regla que se utiliza como referencia de medida de distancia $y$, posteriormente se utiliza como escala cuando se procesa el video. La pantalla puede estar soportada por una base de madera.

- Acelerómetro: sistema de medición de aceleraciones en base a Arduino. El sistema incluye: microprocesador Arduino Nano, acelerómetro digital BMA 180, reloj RTC, un adaptador micro SD donde guarda la información y baterías AA como fuente de alimentación.

- Laser: se utilizó un láser de $5 \mathrm{~mW}$ de potencia de color verde, por tener un espectro de luz visible mayor a los otros colores.

- Pantalla de proyección: pantalla blanca con una regla, soportada por una base de madera. La base sirve de apoyo para un celular utilizado para grabar la proyección del rayo láser sobre la pantalla.

- Celular con cámara: samsung galaxy S7, se eligió por su disponibilidad y grabación de vídeos en 3840 $\times 2160$ pixeles a $30 \mathrm{fps}, 1920 \times 1080$ pixeles a $60 \mathrm{fps}$

- Deformímetro (o comparador): se utilizó deformímetro con precisión de 0.001 pulgadas $(0.0254 \mathrm{~mm})$.

\subsection{Instrumentación}

Para la medición de desplazamientos y aceleraciones se utilizaron los siguientes métodos:

- Método de punto+cámara: El movimiento del puente se registra con la cámara digital, grabando el movimiento de la pantalla de referencia colocada sobre la estructura (en la ubicación de interés). La cámara digital se coloca fuera del puente (donde la vibración del terreno es despreciable). La cámara se coloca paralela a la carretera (aprovechando la acera como área firme, limpia y de fácil acceso), y a la misma altura del punto sobre la pantalla de referencia. La cámara se pega con tape doble contacto a una base de madera que ayuda a brindar un mejor soporte a la misma, ya que los trípodes comerciales económicos no brindan la estabilidad necesaria.

- Método de laser+cámara: El láser se coloca sobre la estructura en el punto de interés y se proyecta sobre la pantalla de proyección. El láser debe quedar fijo a la estructura. Se coloca la pantalla de proyección (con su base) en la acera fuera del puente paralela a la carretera y la misma altura del láser. Se coloca el celular sobre la base de la pantalla de proyección. El movimiento del puente se registra con la cámara de celular, grabando el 
movimiento del punto laser sobre la pantalla de proyección.

- Método utilizando el deformímetro: Se coloca bajo la estructura del puente, soportado por una plataforma inercial apoyada en terreno firme.

- Método utilizando el acelerómetro: Se apoya fijamente sobre la estructura del puente y registra los cambios de aceleración durante el movimiento del puente.

\subsubsection{Instrumentación de pruebas de laboratorio}

Para verificar el desempeño de los métodos fotogramétricos, se utilizaron los métodos de punto+cámara y laser+cámara para medir desplazamientos durante pruebas estáticas y dinámicas en laboratorio. Las pruebas se hicieron sobre un espécimen de madera de $2.45 \mathrm{~m}$ de longitud, con sección de $19 \mathrm{~cm}$ de ancho y $2 \mathrm{~cm}$ de espesor (ver figura $3)$.

Todos los equipos (ver sección 3.1) se colocaron para registrar el movimiento (desplazamiento o aceleración) en la mitad de la luz del espécimen. Para los distintos métodos se instrumentó el espécimen como sigue:

- Método punto+cámara, la pantalla de referencia fue adherida a la parte lateral del espécimen y la cámara se colocó a la misma altura y a $1.5 \mathrm{~m}$ de distancia del espécimen.

- Método laser+cámara, la pantalla de proyección se ubicó a $1.5 \mathrm{~m}$ de distancia del espécimen. El láser se colocó sobre la caja del acelerómetro y se pegó con tape doble contacto para que quedara fijo.

- Método utilizando el deformímetro, el deformímetro se colocó apoyado en el suelo, de forma tal que su punta de medición estaba en contacto con la parte inferior del espécimen.

- Método utilizando el acelerómetro, el acelerómetro se colocó sobre el espécimen y se pegó con tape doble contacto, a la mitad de la luz.

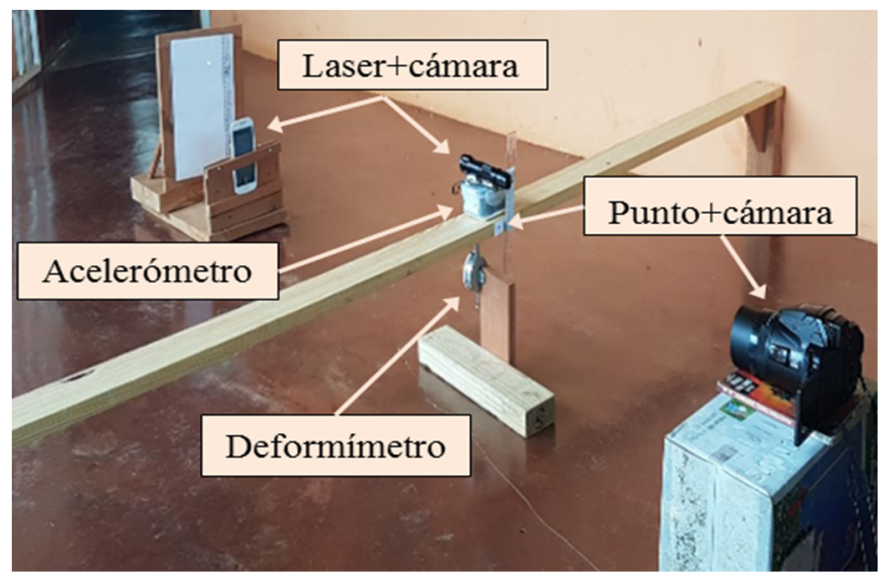

Figura 3. Instrumentación durante pruebas en espécimen.

\subsubsection{Instrumentación en puentes}

Para registrar los desplazamientos verticales de los puentes se colocó la pantalla de referencia a la mitad de la luz y la cámara sobre un apoyo fijo fuera del puente. Junto a la pantalla de referencia colocamos el acelerómetro (ver figura 4). Se registró el movimiento cuando pasaban vehículos pesados sobre los puentes, en intervalos de medición de 30 segundos.

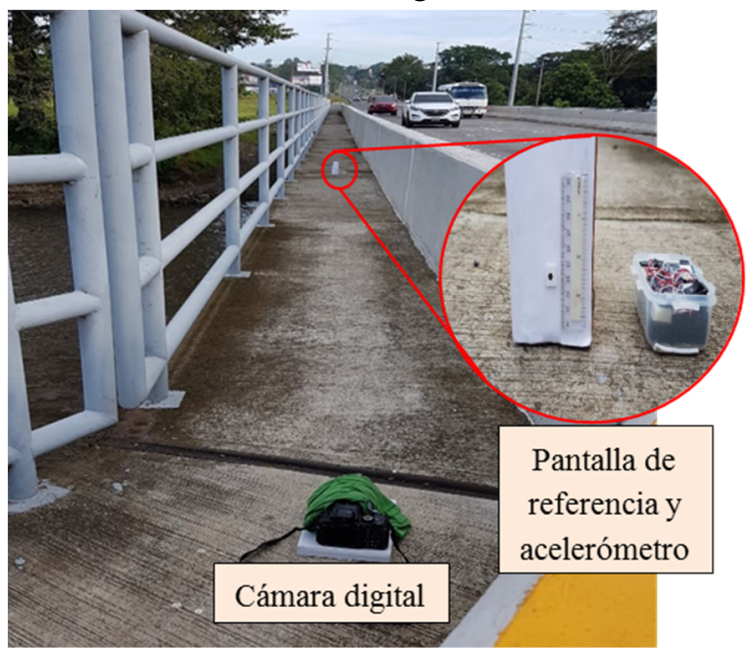

Figura 4. Instrumentación en el puente sobre el Río David aguas abajo, luz 2 (L-344760E-932530N).

Durante la implementación del método en los puentes vehiculares surgieron fuentes de error que no estaban presentes en el laboratorio. Las fuentes de error se determinaron y se aplicaron modificaciones al método para minimizar el efecto de las mismas. A continuación, se mencionan las fuentes de error, lo que causaba y la solución implementada: 
- El vapor generado por la carretera, debido a las altas temperaturas, causaba distorsión en las imágenes al enfocar la pantalla de referencia. La solución fue realizar las mediciones en horas de la mañana cuando las temperaturas eran más baja (aproximadamente hasta las 10:00 am).

- Las corrientes de aire ambientales o generadas por los vehículos, causaban vibraciones en el lente de la cámara transmitiéndoselas a las imágenes. La solución fue cubrir con una tela la parte superior y lateral de la cámara, y pegar la base de madera de la cámara, con tape doble contacto, a la acera.

- El paso de vehículos pesados al lado de la cámara, fuera del puente, causaba vibraciones en la cámara, las cuales quedaban registradas en el video. Estas pequeñas vibraciones no afectaban los desplazamientos máximos que ocurrían cuando el vehículo pesado se encontraba en el puente. Adicionalmente, la vibración inducida en el terreno no afecta la determinación de la frecuencia de los primeros modos de vibración, ya que el puente vibra por mucho más tiempo que el terreno, con amplitudes mucho mayores $\mathrm{y}$ a frecuencias diferentes que las vibraciones causadas por los vehículos en el terreno.

\subsection{Procesamiento}

Para el procesamiento del video obtenido durante las pruebas se utilizó Tracker [19], programa desarrollado por Open Source Physics, el cual permite monitorear el movimiento de un objeto grabado en video, para determinar el desplazamiento, generar tablas y gráficas de desplazamientos de los puentes estudiados (ver figura 5). Para la determinación de las frecuencias de vibración se calculó la transformada rápida de Fourier de los datos (desplazamiento y aceleraciones) utilizando Matlab [20], programa desarrollado por MathWorks, para la solución de problemas matemáticos.

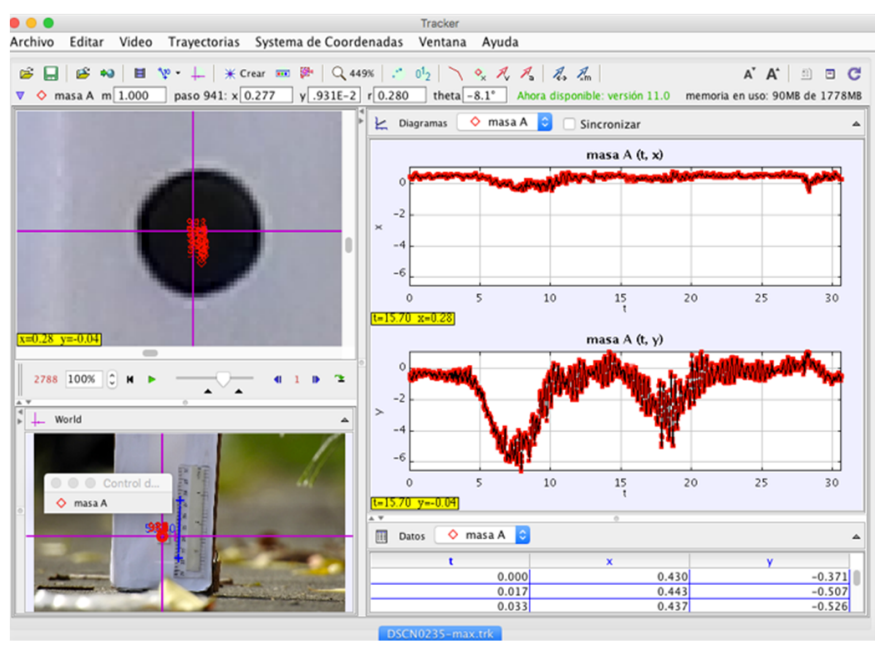

Figura 5. Interfaz del programa Tracker procesando el video del puente sobre la quebrada San Cristóbal aguas abajo (L-340130E-931970N).

\section{Resultados de prueba estática}

Para validar y verificar la precisión de los métodos propuestos, se compararon las mediciones captadas durante la prueba de carga de laboratorio usando el deformímetro versus aquellas captadas mediante los métodos de laser+cámara y punto+cámara. La prueba se realizó a un espécimen de madera (ver figura 6) aplicándole cargas estáticas de $37 \mathrm{~N}$ en dos incrementos (ver figura 7).

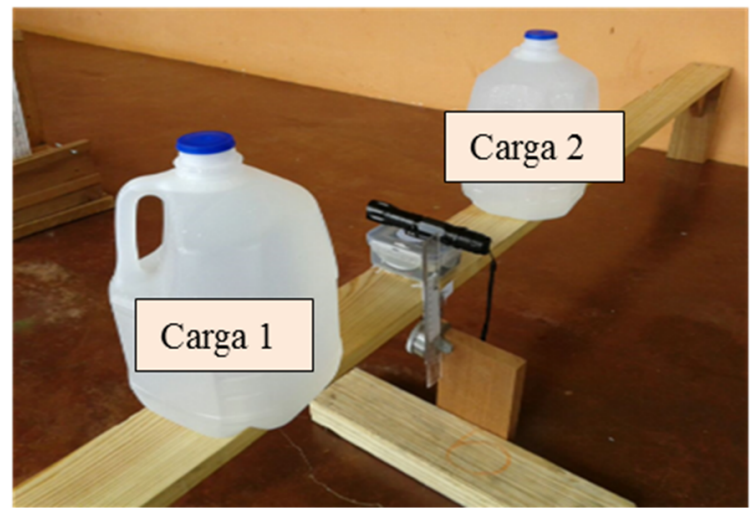

Figura 6. Prueba estática de espécimen de madera.

En los resultados de la prueba estática (ver Tabla 2) se observa que el método de punto+cámara brinda los valores de desplazamiento más cercanos al deformímetro (diferencias menores a $0.1 \mathrm{~mm}$ ), por lo cual fue el método que se implementó en los puentes vehiculares. Mientras que el método de laser se aleja más de los resultados del deformímetro (diferencias en 
el orden de $0.2 \mathrm{~mm}$ ), el error del láser puede estar relacionado con la torsión en el espécimen.

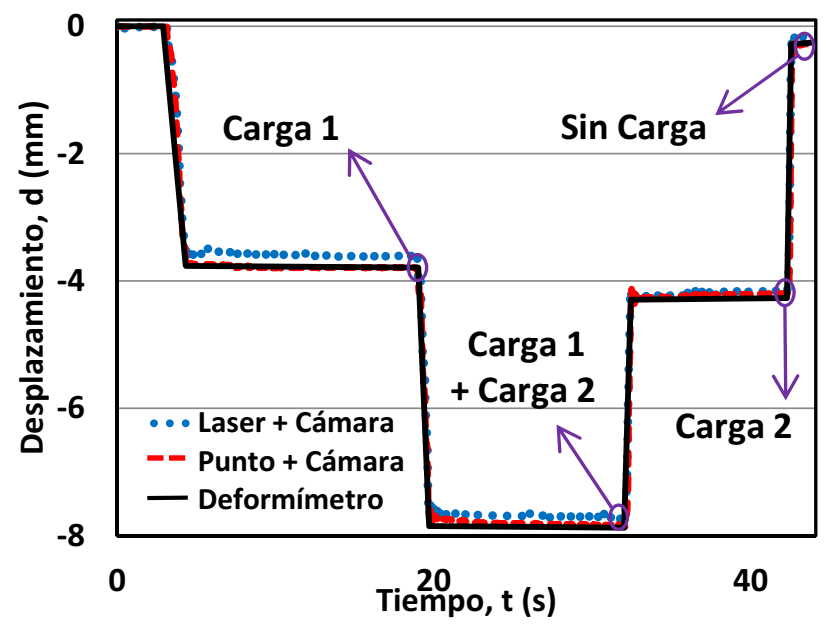

Figura 7. Resultados de prueba estática en espécimen.

Tabla 2. Resultados de la prueba estática en espécimen

\begin{tabular}{|c|c|c|c|c|}
\hline & \multicolumn{4}{|c|}{ Desplazamiento (mm) } \\
\hline Etapa & ש్ & 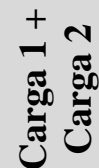 & 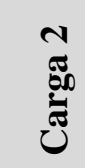 & 䩞 \\
\hline Laser+Cámara & 3.61 & 7.74 & 4.31 & 0.14 \\
\hline Punto+Cámara & 3.80 & 7.86 & 4.22 & 0.25 \\
\hline Deformímetro & 3.78 & 7.87 & 4.25 & 0.25 \\
\hline $\begin{array}{c}\text { Diferencia entre } \\
\text { Laser+Cámara y } \\
\text { Deformímetro }\end{array}$ & 0.17 & 0.13 & 0.06 & 0.11 \\
\hline $\begin{array}{c}\text { Diferencia entre } \\
\text { Punto+Cámara y } \\
\text { Deformímetro }\end{array}$ & 0.02 & 0.01 & 0.03 & 0.00 \\
\hline
\end{tabular}

La mejora del método de laser+cámara escapa del alcance de este artículo, sin embargo, basados en la experiencia de las pruebas, se puede sugerir que el método laser+cámara puede mejorar como sigue:

- El láser debe colocarse de manera fija fuera de la estructura y la pantalla de proyección en el punto de interés en la estructura, para eliminar efectos secundarios relacionados con la rotación (o torsión) del puente.

- Proyectar el rayo láser a través de un telescopio, para reducir el tamaño del punto laser proyectado, lo que elimina la divergencia del haz laser y le da una forma circular definida al punto proyectado [8].

\section{Resultados de prueba dinámica}

Para validar el uso de los métodos durante pruebas dinámicas, se compararon los resultados del acelerómetro, con el método de laser+cámara y con el método de punto+cámara, al darle un golpe al espécimen en el centro de su luz. Posteriormente se determinaron los desplazamientos, aceleraciones y la frecuencia de vibración producto de la respuesta dinámica del espécimen. El acelerómetro está programado para capturar 400 datos por segundos (400 $\mathrm{Hz}$ ) y las cámaras configuradas a grabar a 30 fotos por segundo $(30 \mathrm{~Hz})$.

Los desplazamientos dinámicos obtenidos con los métodos fotogramétricos de laser+cámara y punto+cámara son muy parecidos. Por otro lado, cuando se integran los datos crudos del acelerómetro (sin usar ninguna corrección), los desplazamientos calculados se desvían rápidamente de los desplazamientos verdaderos (ver figura 8).

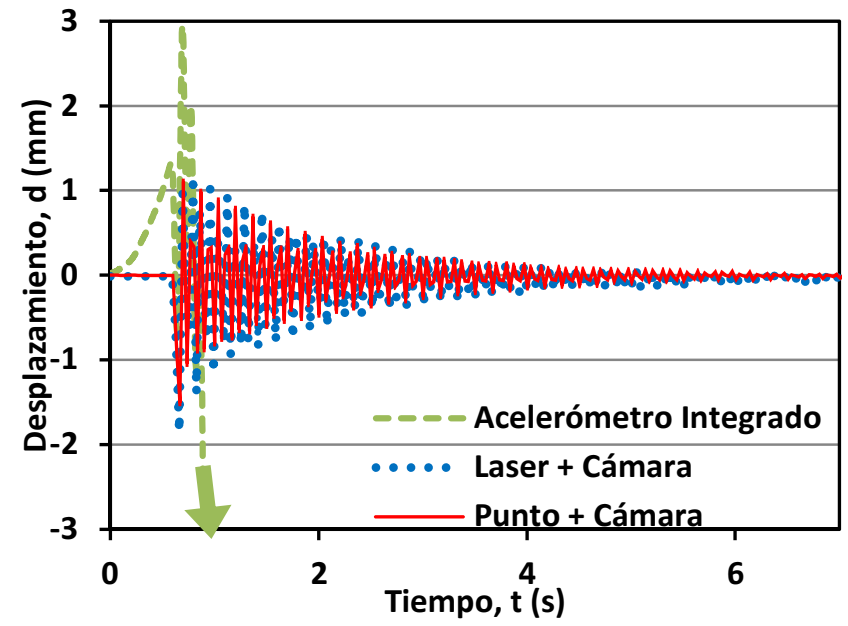

Figura 8. Resultados de desplazamiento de prueba dinámica en espécimen.

Al derivar con respecto al tiempo las historias de desplazamiento, captadas mediante los métodos de laser+cámara y punto+cámara, se obtienen acelerográmas similares a aquellos captados por el acelerómetro BMA180 (ver figura 9), el cual tiene alta precisión $(0.25 \mathrm{mg})$ [21].

Los acelerómetros han sido ampliamente utilizados para el estudio de frecuencias de vibración de puentes $[3,22,23]$, por lo que en este estudio sus mediciones serán consideradas como adecuadas. Las frecuencias obtenidas al analizar los datos de desplazamiento 
obtenidos de los métodos de laser+cámara y punto+cámara presentan diferencias de $0.02 \mathrm{~Hz}$ o menos con respecto a aquellas obtenidas del análisis de la data del acelerómetro (ver figura 10).

Considerando todo lo anterior, se propone el método de punto+cámara para la medición de desplazamientos estáticos y dinámicos (y frecuencias de vibración) en puentes en uso.

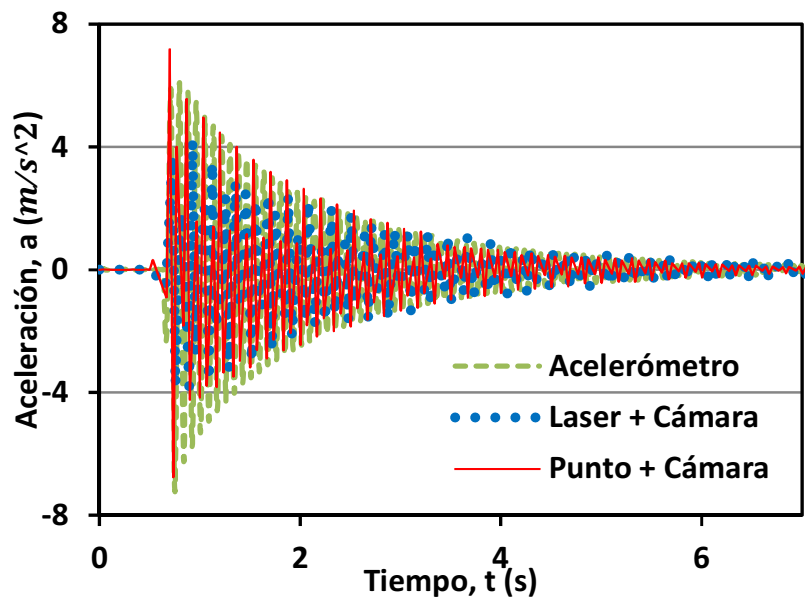

Figura 9. Resultados de aceleración de prueba dinámica en espécimen.

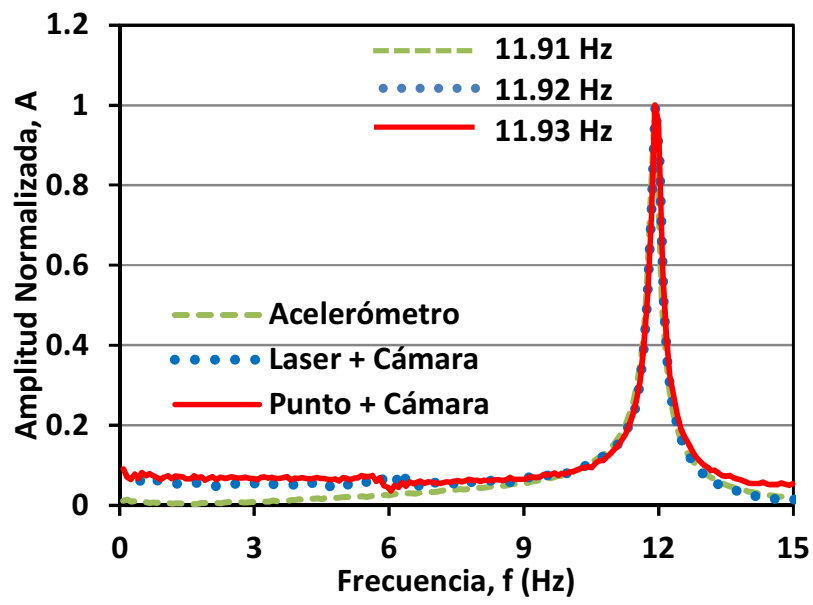

Figura 10. Resultados de frecuencia de vibración de prueba dinámica en espécimen.

\section{Resultados de mediciones en puentes}

A continuación, se presentan los resultados de las mediciones realizadas en puentes vehiculares en uso utilizando el método de punto+cámara (ver figura 11 a figura 15). En las figuras se puede observar que cuando las cargas pasan sobre el puente, este se deforma alejando sus oscilaciones del valor medio.

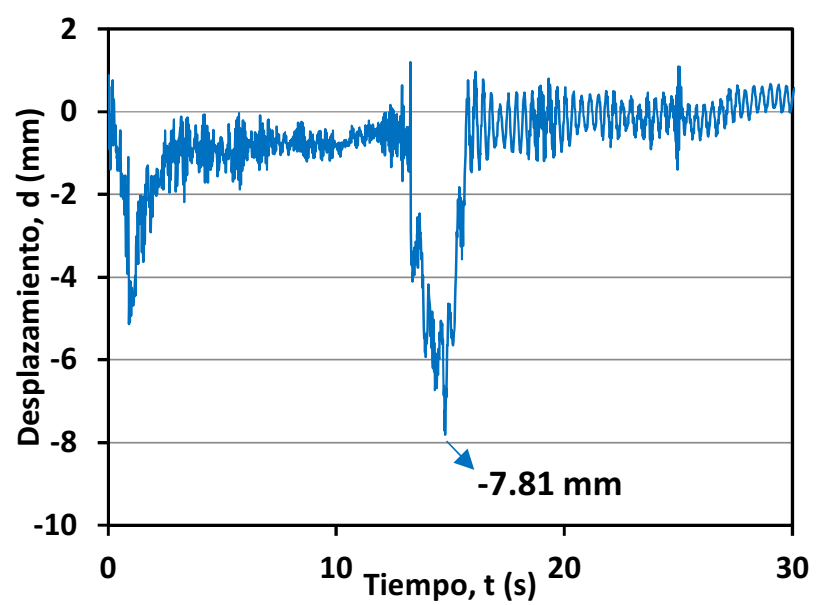

Figura 11. Desplazamiento dinámico registrado en el puente sobre el río Chirigagua (L-330300E-936810N).

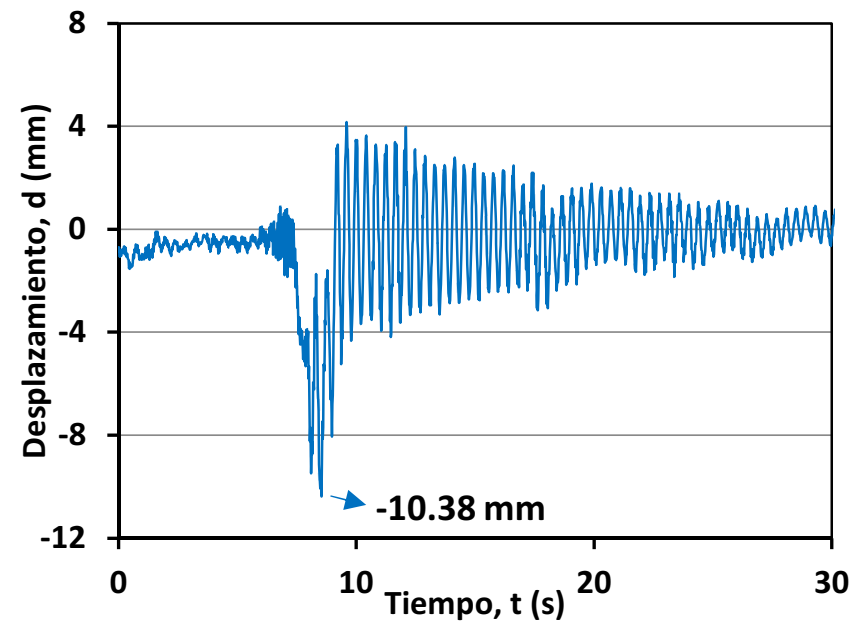

Figura 12. Desplazamiento dinámico registrado del puente sobre el Río Platanal (L-332610E-935900N).

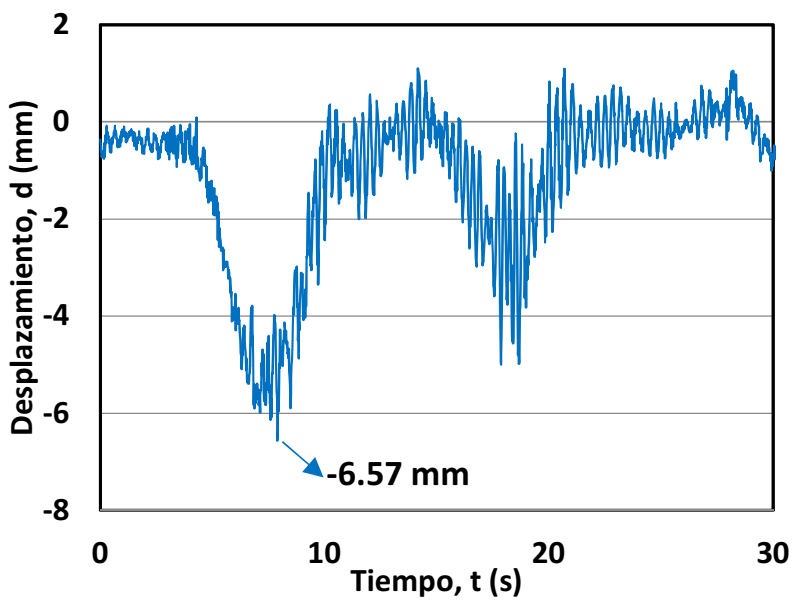

Figura 13. Desplazamiento dinámico registrado en el puente sobre la quebrada San Cristóbal (L-340130E-931970N). 


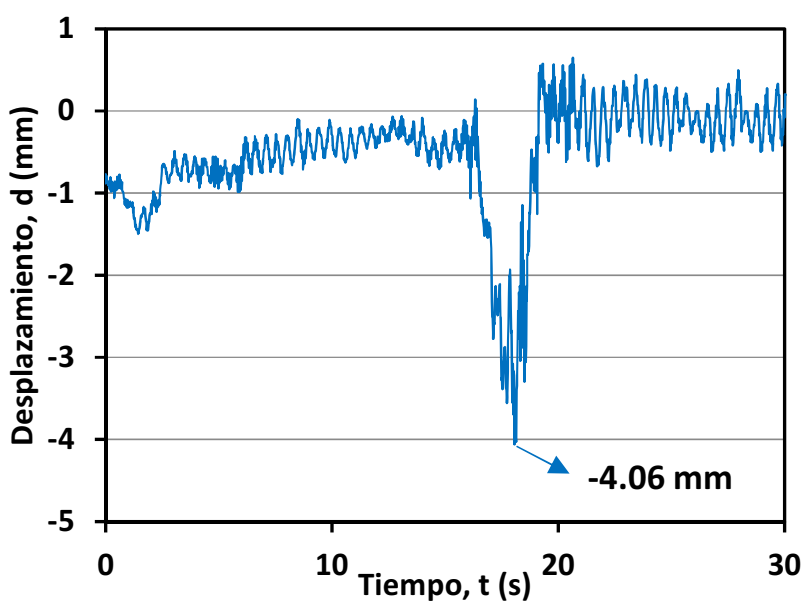

Figura 14. Desplazamiento dinámico registrado en el puente sobre el río David, luz 1(L-344740E-932560N).

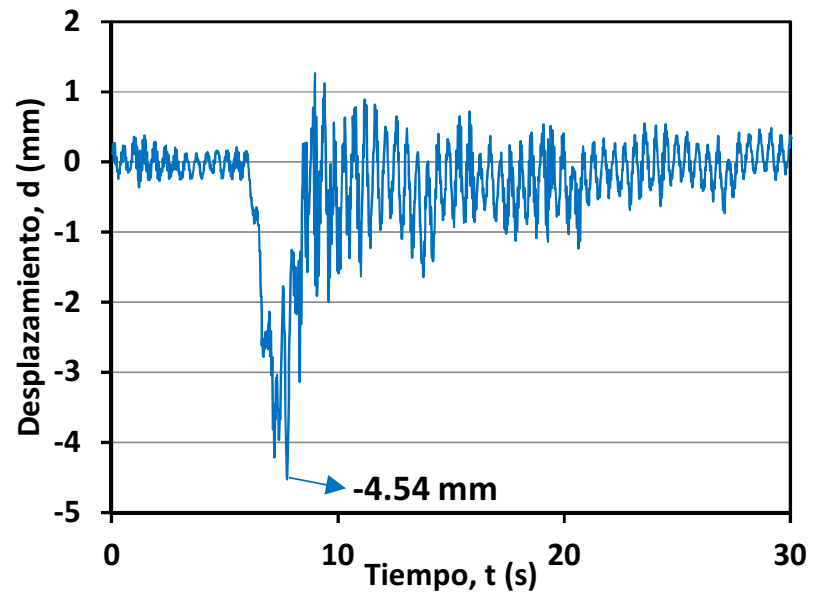

Figura 15. Desplazamiento dinámico registrado en el puente sobre el Río David, luz 2 (L-344760E-932530N).

Teniendo los datos de desplazamiento dinámico y aceleración para el mismo intervalo de medición, de cada puente estudiado, se calculó los contenidos de frecuencias, y la frecuencia natural del primer modo de vibración.

Como se pude ver desde la figura 16 a la figura 20, las frecuencias obtenidas con el acelerómetro y las obtenidas con el método de punto+cámara son prácticamente iguales, observándose variaciones en el segundo decimal, lo cual confirma que el método de punto+cámara se podría utilizar para determinar las frecuencias de vibración en puentes con gran precisión.

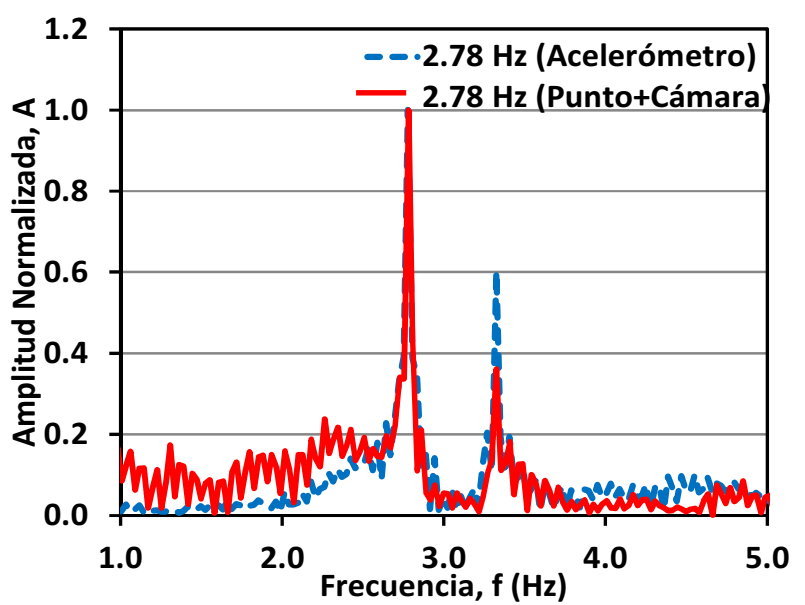

Figura 16. Contenido de frecuencia del puente sobre el Río Chirigagua (L-330300E-936810N).

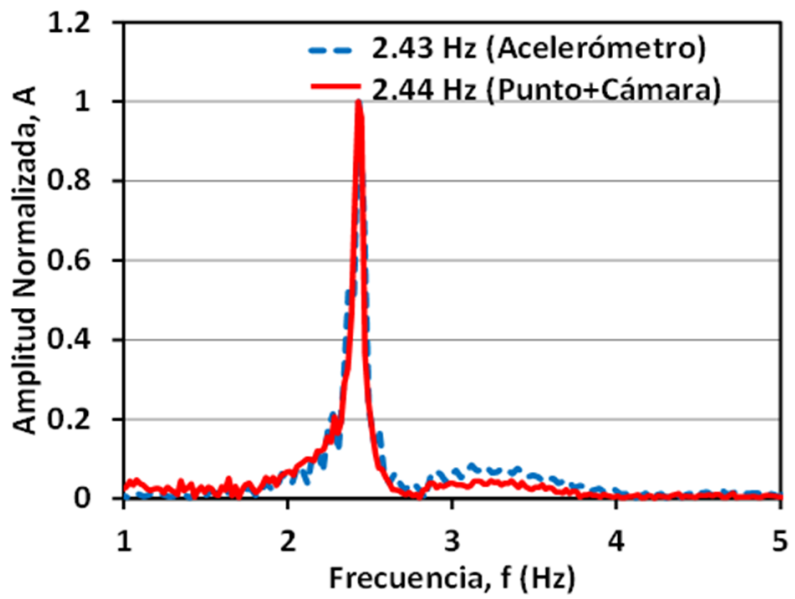

Figura 17. Contenido de frecuencia del puente sobre el Río Platanal (L-332610E-935900N).

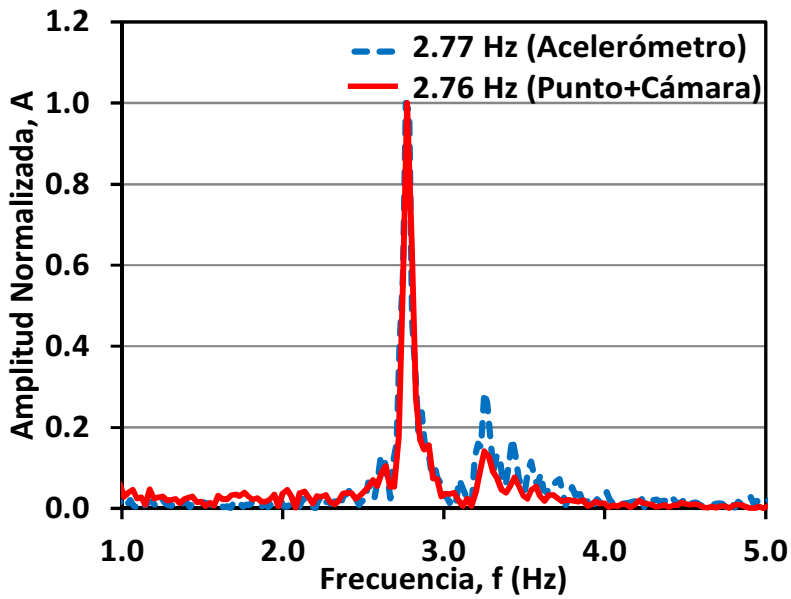

Figura 18. Contenido de frecuencia del puente sobre la Quebrada San Cristóbal (L-340130E-931970N). 


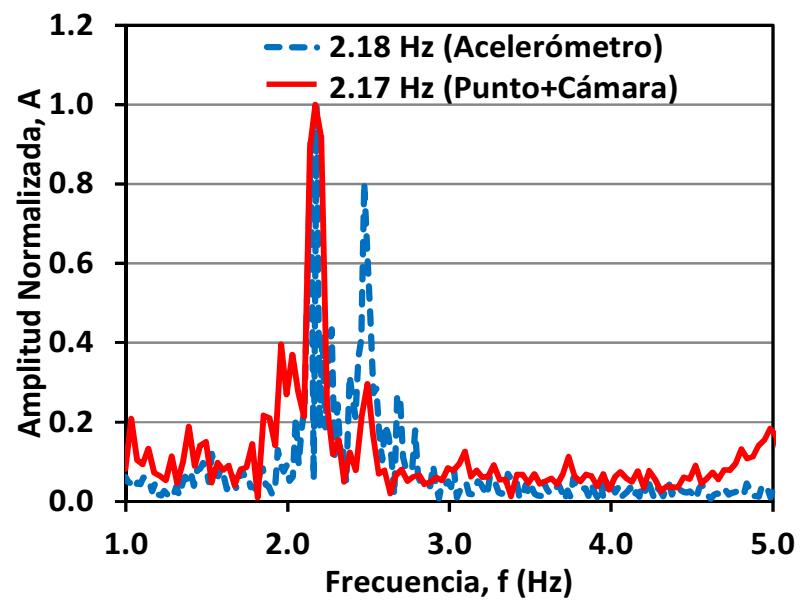

Figura 19. Contenido de frecuencia del puente sobre el Río David, luz 1 (L-344740E-932560N).

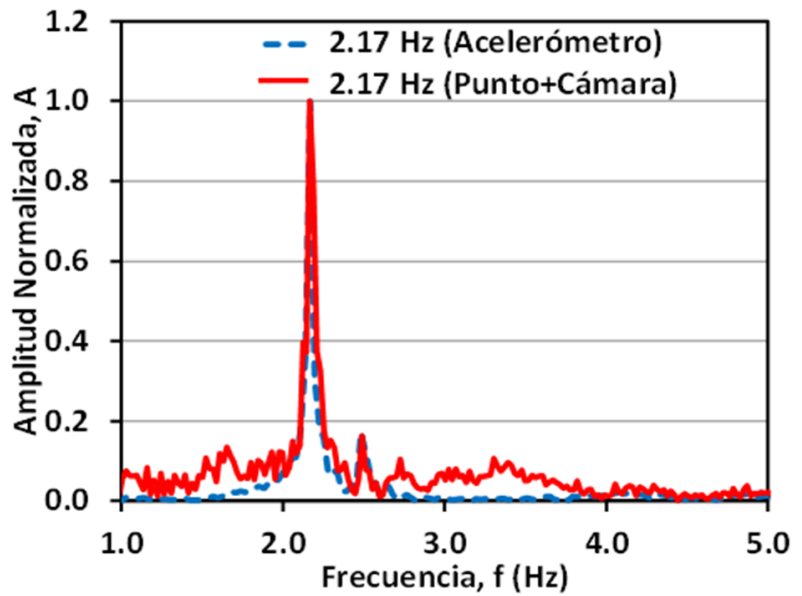

Figura 20. Contenido de frecuencia del puente sobre el Río David, luz (L-344760E-932530N).

En la tabla 3, se muestran las longitudes, frecuencias y periodos obtenidas con el método punto+cámara en los puentes estudiados y se calcula una relación con el periodo esperado, para ver qué tanto se acerca al valor esperado. En este estudio se adoptará la ecuación:

$$
T e=1 \mathrm{~s}\left(\frac{\mathrm{L}}{100 \mathrm{~m}}\right)
$$

Donde Te es el período esperado y L la longitud de la luz [3].

Considerando que la frecuencia de vibración está relacionada con las características del puente, las frecuencias (o periodos) captadas durante este estudio pueden considerarse representativas de los puentes estudiados. En este sentido, se puede observar que los periodos de vibración fundamental son similares a los periodos esperados, lo cual indica que la relación entre la masa, la frecuencia y la luz de los puentes estudiados cae dentro de los rangos normales [3].

Tabla 3. Resultados de frecuencia y período de vibración

\begin{tabular}{|c|c|c|c|c|c|}
\hline 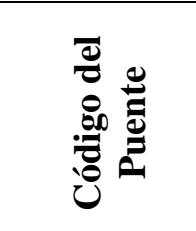 & 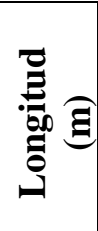 & 苞 & 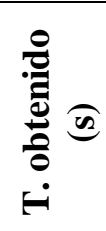 & 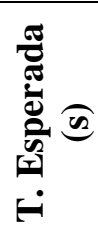 & 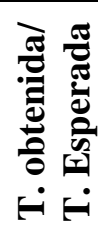 \\
\hline $\begin{array}{l}\text { L-330300E- } \\
936810 \mathrm{~N}\end{array}$ & 35.4 & 2.78 & 0.36 & 0.35 & 1.03 \\
\hline $\begin{array}{l}\text { L-332610E- } \\
935900 \mathrm{~N}\end{array}$ & 38.6 & 2.44 & 0.41 & 0.39 & 0.95 \\
\hline $\begin{array}{l}\text { L-340130E- } \\
931970 \mathrm{~N}\end{array}$ & 35.6 & 2.76 & 0.36 & 0.36 & 1.00 \\
\hline $\begin{array}{l}\text { L-344740E- } \\
932560 \mathrm{~N}\end{array}$ & 42.0 & 2.17 & 0.46 & 0.42 & 1.10 \\
\hline $\begin{array}{l}\text { L-344760E- } \\
932530 \mathrm{~N}\end{array}$ & 42.0 & 2.17 & 0.46 & 0.42 & 1.10 \\
\hline
\end{tabular}

En la tabla 4, se muestra un resumen de los desplazamientos máximos obtenidos en cada puente y su aceleración máxima correspondiente, junto con el desplazamiento teórico máximo que debe cumplir según la normativa AASHTO LRFD. Como se puede ver los desplazamientos obtenidos están muy por debajo de los permitidos durante las pruebas de carga.

Tabla 4. Resultados de desplazamiento y aceleración

\begin{tabular}{|c|c|c|c|c|}
\hline Código & 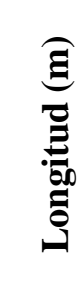 & 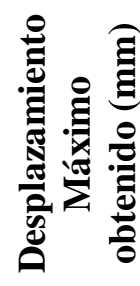 & 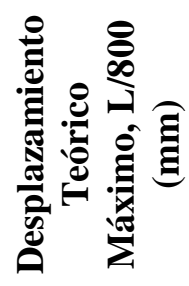 & 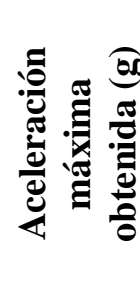 \\
\hline $\begin{array}{c}\text { L-330300E- } \\
936810 \mathrm{~N}\end{array}$ & 35.4 & -7.81 & -44.25 & -0.074 \\
\hline $\begin{array}{c}\text { L-332610E- } \\
935900 \mathrm{~N}\end{array}$ & 38.6 & -10.38 & -48.25 & -0.134 \\
\hline $\begin{array}{c}\text { L-340130E- } \\
931970 \mathrm{~N}\end{array}$ & 35.6 & -6.57 & -44.50 & -0.083 \\
\hline $\begin{array}{c}\text { L-344740E- } \\
932560 \mathrm{~N}\end{array}$ & 42.0 & -4.06 & -52.5 & -0.039 \\
\hline $\begin{array}{c}\text { L-344760E- } \\
932530 \mathrm{~N}\end{array}$ & 42.0 & -4.54 & -52.5 & -0.047 \\
\hline
\end{tabular}


El objetivo de este artículo no es verificar la condición estructural de los puentes, por lo que no se deben tomar los desplazamientos máximos medidos como una referencia del estado del puente. Los desplazamientos fueron presentados en este artículo con el objetivo de ilustrar los resultados que se obtienen del método, y son representativos de la respuesta ante el tráfico existente al momento de la prueba.

\section{Conclusiones}

Basado en los resultados de este estudio se puede concluir que el método de punto+cámara:

1. Permite medir desplazamientos verticales $\mathrm{y}$ horizontales, estáticos y dinámicos, así como la frecuencia de vibración.

2. Ofrece gran precisión en la medición de desplazamientos (por medio de las pruebas de laboratorio se observó que el método punto+cámara tiene una precisión en el orden de $0.1 \mathrm{~mm}$ ).

3. Permite el cálculo de la frecuencia de vibración principal con gran precisión (en el orden de $0.01 \mathrm{~Hz}$ )

4. Es de configuración fácil y rápida

5. Es de bajo costo para puentes de mediana longitud (ya que se pueden usar cámaras digitales no profesionales).

\section{Recomendaciones}

Basado en los resultados de este estudio se puede recomendar:

1. Utilizar el método de punto+cámara para medir los desplazamientos de puentes bajo tráfico vehicular normal y durante pruebas de carga estáticas.

2. Utilizar el método de punto+cámara para pruebas de laboratorio en vigas y para cualquier prueba donde se desee conocer el desplazamiento estático y/o dinámico de una estructura al aplicarle carga.

3. Estudiar la posibilidad de realizar pruebas de carga dinámica utilizando el método de punto+cámara y camiones con peso conocido que transiten por el puente a velocidades bajas, pero sin detenerse, cerrando el tráfico solamente durante el tiempo que tome a los camiones atravesar el puente. Este tipo de prueba reduciría el tiempo de cierre del puente y permitiría conocer la respuesta del puente ante cargas conocidas.

4. Realizar un estudio para verificar la factibilidad de utilizar en nuestro país métodos fotogramétricos, utilizando cámaras no-profesionales, en puentes de gran longitud utilizando pantallas de referencia de gran tamaño, o elementos del puente como punto de referencia.

\section{Referencias}

[1] J. A. Marulanda, J. C. Marulanda, P. Thomson, "Monitoreo de Salud Estructural", Ingeniería y Competitividad, Vol. 2, Diciembre 2000.

[2] Moreno Claudia P., Thomson Peter, "Incertidumbre paramétrica en modelos dinámicos de estructuras civiles", Universidad del Valle, Cali, Colombia, Ingeniería y Competitividad, vol. 12, núm. 1, 2010, pp. 111-125.

[3] S. M. Rodríguez, J. M. Gallardo, O. A. Araúz, "Estudio del período de vibración de puentes de concreto en Panamá", Grupo de investigación: Salud Estructural de Puentes, Universidad Tecnológica de Panamá, 2017.

[4] Albert, J., H.-G. Maas, A. Schade, W. Schwarz, Pilot studies on photogrammetric bridge deformation measurement, Proceedings of the 2nd IAG Commission IV Symposium on Geodesy for Geotechnical and Structural Engineering, Berlin, Germany, 21-24 May, 2002.

[5] Juan M. Caicedo, "Displacement Measurements in Civil Structures Using Digital Cameras and Lasers", University of South Carolina, Department of Civil and Environmental Engineering, 2005.

[6] Jónatas Valença, Eduardo Júlio, Helder Araújo, “Application of photogrammetry to bridge monitoring", University of Coimbra, Portugal, 2008.

[7] J. M. Franco, J. H. Murcia, D. Gómez, P. Thomson, "Implementación de sensores láser para medir desplazamientos en estructuras", Grupo de Investigación en Ingeniería Sísmica, Ingeniería Eólica y Estructuras Inteligentes, G-7, Universidad del Valle, Cali, Colombia, 2008.

[8] J. M. Franco, D. Gómez, J. H. Murcia, P. Thomson, J. Marulanda, "Desarrollo e implementación de un sistema láser para caracterización dinámica y medición de desplazamientos en estructuras civiles", Grupo de Investigación en Ingeniería Sísmica, Ingeniería Eólica y Estructuras Inteligentes, G-7, Universidad del Valle, Rev. Fac. Ing. Univ. Antioquia N. ${ }^{\circ} 60$ pp. 170-181. Septiembre, 2011.

[9] Ö. AVŞAR, D. AKCA y O. ALTAN, "Photogrammetric Deformation Monitoring of the Second Bosphorus Bridge in Istanbul", The International Archives of the Photogrammetry, Remote Sensing and Spatial Information Sciences, Volume XL-5, Istanbul Technical University, Istanbul, 2014.

[10] Xuefeng Zhao ,Hao Liu, Yan Yu, Xiaodong Xu, Weitong Hu, Mingchu Li, Jingping Ou, "Bridge Displacement Monitoring Method Based on Laser Projection-Sensing Technology", Dalian University of Technology, Dalian 116000, China, 2015.

[11] ASHIMA SETIA, DR. S. BHALLA, "Photogrammetry for Non-conact Measurement of Deflection", Department of Civil Engineering, Indian Institute of Technology Delhi, April 2010.

[12] Ireneusz WYCZAŁEK, Michał WYCZAŁEK, "The Potential of Photogrammetric Method of Measurement Dynamic Displacements of Flexible Bridges".

[13] Palazzo, D.; Friedmann, R.; Nadal, C.; Santos, F.M.; Veiga, L.; Faggion, P., "Dynamic monitoring ofstructures using a robotic total station", 2006. 
[14] AASHTO LRFD Bridge Design Specifications, 2007. Cuarta Edición. AASHTO, Washington, D.C.

[15] Daniel Abalo C., "Reloj comparador", Tecnología de comparación.

[16] MARVIN A.CARDOZA, JOSÉ E. VILLALOBOS Z., "Evaluación estructural de un puente mediante la realización de una prueba de carga estática", Universidad de El Salvador, Ciudad Universitaria, 2005.

[17] Rosendo R. Andrade, Carlos R. Moraila, Pedro E. Ortíz, "Medición de la vibración en estructuras de puentes empleando GPS", Universidad Autónoma de Sinaloa, 2012.

[18] Diego González A., Javier Gómez L., José Sánchez A. "New Approach for Structural Monitoring of Large Dams with a Three-Dimensional Laser Scanner", Cartographic and Land Engineering Department, University of Salamanca, 2008.

[19] Tracker Video Analysis and Modeling Tool for Physics Education. [Online]. Available: http://physlets.org/tracker/.

[20] "MATLAB Student", [Online]. Available: https://www.mathworks.com/academia/student-version-b.html.

[21] "BMA180 Digital Triaxial Acceleration Sensor", Data Sheet, [Online].

Available: http://irtfweb.ifa.hawaii.edu/ tcs3/jumpman/ jumppc/1107BMA180/BMA180-DataSheet-v2.5.pdf

[22] Alejandro de Miguel T., Pablo M., "ANÁLISIS DINÁMICO DE ESTRUCTURAS EN EL DOMINIO DE LA FRECUENCIA", Escuela Técnica Superior de Ingenieros de Caminos, Canales y Puertos, Madrid, Junio de 2011.

[23] J. Valdés, J. De la Colina, "Análisis de la Amplificación Dinámica de la Carga Viva en Puentes con Base en Pruebas Experimentales", Facultad de Ingeniería, Universidad Autónoma del Estado de México, Revista Tecnológica ESPOL, Vol. 21, N. 1, 149-156, Octubre, 2008. 